\title{
Disruption of Pharmacotherapy During the Transition from Adolescence to Early Adulthood in Patients with Attention-Deficit/Hyperactivity Disorder: A Claims Database Analysis Across the USA
}

\author{
Sepehr Farahbakhshian ${ }^{1}$ (D) Rajeev Ayyagari ${ }^{2} \cdot$ Daniel S. Barczak $^{1} \cdot$ Simerpal K. Gill $^{3} \cdot$ Wenxi Tang $^{4}$. \\ Thomas Kulalert $^{4} \cdot$ Madeline Jenkins $^{4} \cdot$ William Spalding $^{1}$
}

Accepted: 17 March 2021 / Published online: 15 April 2021

(c) The Author(s) 2021

\begin{abstract}
Background and Objective Attention-deficit/hyperactivity disorder (ADHD) treatment rates in adults are low, possibly owing to discontinuation of pediatric care due to various circumstances (including inadequate health insurance coverage, poor disease insight, and patient/family resistance, as well as those who manage their ADHD independent of pharmacologic intervention) during the transition from adolescence to adulthood. To improve the understanding of treatment patterns during this transition, this study characterized pharmacotherapy use in patients with ADHD aged 16-21 years.

Methods A retrospective claims analysis of the $\mathrm{IBM}^{\circledR}$ MarketScan ${ }^{\circledR}$ Commercial Databases, which represent all census regions of the USA, included patients aged 16-21 years with two or more ADHD diagnoses between 1/1/2008 and 12/31/2017 (one or more diagnoses during the year of age 17) who were continuously enrolled from ages 16-21 years and prescribed ADHD medication for $\geq 6$ months at age 17 years. Pharmacotherapy use was assessed longitudinally. Comparisons between ages were conducted using Wilcoxon signed-rank tests and McNemar tests. Treatment discontinuation was estimated using Kaplan-Meier analyses.

Results The analysis included 10,292 patients. The overall percentage of patients receiving pharmacotherapy significantly decreased ( $p<0.001$, regardless of treatment type and presence of co-occurring psychiatric disorders) as patients aged, with a median time to treatment discontinuation of 2.94 years. Among patients using pharmacotherapy at the age of 17 years, more than $30 \%$ were no longer using pharmacotherapy at age 21 years. As patients aged, the percentage using longacting amphetamines or methylphenidates decreased, and the percentage receiving no treatment increased. The percentage of patients with disrupted treatment from age 18 to 21 years ranged from 17.9 to $24.1 \%$. After transitioning to disrupted treatment or no treatment, low percentages of patients returned to pharmacotherapy use (disrupted treatment: 15.7-21.5\% per year; no treatment, $2.7-3.8 \%$ per year). Across all age groups, statistically significantly greater $(p<0.05)$ percentages of patients with co-occurring psychiatric disorders used lisdexamfetamine, dextroamphetamine-amphetamine mix short acting, and non-stimulants compared with patients without co-occurring psychiatric disorders. Patients with co-occurring psychiatric disorders remained on ADHD pharmacotherapy longer and switched or augmented their pharmacotherapy more frequently than patients without co-occurring psychiatric comorbidities.

Conclusions Patients rarely reinitiated treatment after pharmacotherapy was disrupted or discontinued, emphasizing the need for increased focus on the management of ADHD as patients transition from adolescence to adulthood.
\end{abstract}

Sepehr Farahbakhshian sep.farah@takeda.com

1 Takeda Pharmaceuticals USA, 95 Hayden Ave, Lexington, MA 02421, USA

2 Analysis Group, Boston, MA, USA

3 Takeda Canada Inc., Toronto, ON, Canada

4 Analysis Group, London, UK 


\section{Key Points}

Among patients diagnosed with attention-deficit/hyperactivity disorder (ADHD) and using ADHD pharmacotherapy at the age of 17 years, more than $30 \%$ of patients were no longer using ADHD pharmacotherapy at the age of 21 years.

Patients who experienced a disruption in ADHD pharmacotherapy or who discontinued ADHD pharmacotherapy rarely reinitiated treatment, emphasizing the need to closely monitor and manage patients with ADHD as they transition from adolescence to adulthood.

Patients with ADHD and other co-occurring psychiatric disorders remained on ADHD pharmacotherapy for longer periods, with greater healthcare resource utilization, and switched and augmented their therapy more often than patients without co-occurring psychiatric disorders.

\section{Introduction}

Attention-deficit/hyperactivity disorder (ADHD) is among the most common neurodevelopmental disorders of childhood [1], with an estimated worldwide prevalence of 7.2\% [2]. However, ADHD is not limited to pediatric populations and has been reported to persist into adulthood in approximately $35-50 \%$ of individuals diagnosed as children [3-5].

Evidence indicates that individuals diagnosed with ADHD exhibit both structural and neurochemical alterations in the brain compared with those not diagnosed with ADHD [6-8]. Pharmacotherapies for ADHD, which alter neurochemical activity in brain regions implicated in the etiology of ADHD [9], are the mainstay of treatment in individuals diagnosed with ADHD [10-12]. In pediatric and adult populations, meta-analyses indicate that stimulants and non-stimulants are more efficacious than placebo in reducing the core symptoms of ADHD [13]. It has been shown that patients with ADHD adherent to their ADHD medication have a significant decrease in unintentional physical injuries and motor vehicle accidents, particularly among male patients with ADHD $[14,15]$. Although treatment rates in the USA have risen since 2001-2003 (approximately 11\%), as recently as 2013, approximately half of adults diagnosed with ADHD were not receiving any treatment [16-18]. Furthermore, although drug adherence and persistence are generally poor among patients with ADHD [19], there are limited data on the disruption of care and its clinical outcome during the transition period from adolescence to young adulthood.
Several factors might contribute to the relatively low rate of treatment in adults with diagnosed ADHD. First, young adults with ADHD are vulnerable to treatment discontinuation because of difficulties associated with the transition from adolescence to adulthood. In a study of college freshmen diagnosed with ADHD, an inability to manage ADHD in the context of living independently was observed, with many participants not adhering to prescribed treatment regimens [20]. An inability to adequately deal with decreased familial support and increased autonomy during the transition from adolescence may contribute to a range of negative educational, occupational, and emotional outcomes reported in adults with ADHD [21]. In an analysis of data from the Multimodal Treatment Study of Children with ADHD, adults diagnosed with ADHD as children had lower education attainment, held jobs for shorter periods and were fired more often, and scored worse on ratings of impulsivity/emotional lability and neuroticism compared with those without a diagnosis of ADHD [21]. Gaps in healthcare may also arise during the transition from pediatric to adult services [22, 23]. In a study of young people aged $14-24$ years, $45 \%$ of participants were not in contact with clinical services, and only $9 \%$ reported having transferred to adult care or preparing for the transition to adult healthcare services despite the fact that a high percentage of participants $(62 \%)$ met criteria for ADHD [22]. This lapse in healthcare could account for the decreased frequency of ADHD pharmacotherapy use that is observed as patients move from adolescence to young adulthood [24].

Individuals diagnosed with ADHD are often diagnosed with co-occurring psychiatric disorders including mood, anxiety, and substance use disorders [16, 25-28]. The presence of co-occurring psychiatric disorders not only complicates the diagnosis and treatment of ADHD [29] but may also influence the maintenance of ADHD treatment [30]. Furthermore, healthcare resource utilization (HRU) and healthcare costs for individuals with ADHD are greater among those diagnosed with ADHD than among individuals without an ADHD diagnosis [25, 28, 31], with the presence of a co-occurring psychiatric disorder further increasing HRU and costs [25, 32].

An increased understanding of ADHD treatment patterns and HRU during the transition from adolescence to adulthood, and of the differences in treatment patterns associated with the presence of psychiatric comorbidities, will further enhance the knowledge of healthcare gaps in young adults diagnosed with ADHD. Therefore, the objectives of this study were to characterize pharmacotherapy treatment patterns and HRU in patients with ADHD overall and as a function of the presence or absence of co-occurring psychiatric disorders, during the transition period from adolescence (age 16 years) to early adulthood (age 21 years). 


\section{Methods}

\subsection{Study Design}

This retrospective study used data from the IBM $^{\circledR}$ MarketScan ${ }^{\circledR}$ Commercial Databases (Somers, NY, USA; formerly the Truven Health MarketScan ${ }^{\circledR}$ Databases) from 1 January, 2008, to 31 December, 2017. These databases include individuals aged $\leq 65$ years, represent all census regions of the USA, and have claims data for medical (provider and institutional) and pharmacy services. Disease diagnosis codes and procedure codes in the databases are based on the International Classification of Diseases, Ninth and Tenth Revisions, Clinical Modifications (ICD-9-CM and ICD-10-CM), the healthcare common procedure coding system, and current procedural terminology codes.

The study design (Fig. 1) included a study period that covered the entire duration over which claims data were obtained. The index date was defined as the month when patients reached 18 years of age. The outcomes period was defined as the period from the index date up to the end of continuous eligibility or data availability.

This study did not involve prospective data collection. Therefore, institutional review board approval and informed consent were not required. Patient data were deidentified in accordance with the patient confidentiality requirements of the Health Insurance Portability and Accountability Act of 1996. A summary of the final study report was provided to the appropriate regulatory bodies in compliance with appropriate guidelines.

\subsection{Patients}

Study inclusion and exclusion criteria are summarized in Fig. 1. To ensure patients had active ADHD, the study included patients with two or more claims with an ADHD diagnosis (ICD-9-CM code 314.0x, ICD-10-CM code F90.x) on different dates and with one or more diagnoses during the year of age 17. Patients were also required to have been prescribed ADHD medication for $\geq 6$ months at age 17 years to ensure that they were actively being treated. Continuous enrollment from age 16 years to early adulthood (i.e., age 21 years) was required to ensure that complete information on each patient was obtained. Patients were excluded if they had any claim with a schizophrenia diagnosis (ICD-9-CM code 295.xx, ICD-10-CM code F20.x) during the entire database period.

\subsection{Measures}

Demographic characteristics (sex, geographic region, insurance type) as of the index date were recorded. Medical and psychiatric disorders during the year before the patient's 18 th birthday were identified using ICD-9 or ICD-10 codes. Identified medical disorders were used to calculate a Charlson Comorbidity Index based on algorithms described by Quan et al. [33].

Attention-deficit/hyperactivity disorder pharmacotherapy use was evaluated by examining treatment discontinuation, switching, augmentation, and sequencing. Treatment discontinuation was defined as a lapse of $>60$ or $>90$ days between the end of medication supply and the next prescription refill for any ADHD medication, with the date of discontinuation being the date of the end of the medication supply. Treatment switching was defined as the initiation of a new ADHD medication that was not part of the index treatment (i.e., the treatment or combination of treatments used as of the patient's 18th birthday) within 60 days before or after the discontinuation of the index treatment. Treatment augmentation was defined as the initiation of a new ADHD medication, or other relevant medication, that was not part of the index treatment before the discontinuation of the index treatment among patients who persisted on the index treatment for $\geq 60$ days. The augmentation treatment was required to overlap with the index treatment for $\geq 60$ days. Discontinuation, switching, and augmentation were evaluated among patients using ADHD pharmacotherapy as of their 18th birthday.

Treatment sequencing for ADHD pharmacotherapies was evaluated in patients aged 17-21 years. Drug class categories were long-acting (LA) amphetamines (dextroamphetamine LA, dextroamphetamine-amphetamine mixed salts LA, lisdexamfetamine), short-acting (SA) amphetamines (dextroamphetamine SA, dextroamphetamine-amphetamine mixed salts SA), LA methylphenidates (methylphenidate LA, dexmethylphenidate LA), SA methylphenidates (methylphenidate SA, dexmethylphenidate SA), and non-stimulants (atomoxetine, clonidine, guanfacine, bupropion). Patients were required to have $\geq 6$ months of medication supply for one or more drug class to be categorized into a specific class, with the selected drug class being the one with the most days of supply. Patients were categorized as using mixed therapy if they had $\geq 6$ months of medication supply, but not for any individual drug class, and as using combination therapy if they had $\geq 6$ months of supply for one or more drug class and multiple drug classes had the same number of days of supply. Patients were categorized as having no treatment if they had no medication supply and as disrupted treatment if they had $<6$ months of medication supply across all drug classes.

Evaluations of HRU included all-cause inpatient visits, all-cause emergency department (ED) visits, and outpatient visits. Outpatient visits included visits to primary care physicians (PCPs), pediatricians, or psychiatrists; they were categorized as being related to ADHD or mood disorders. 


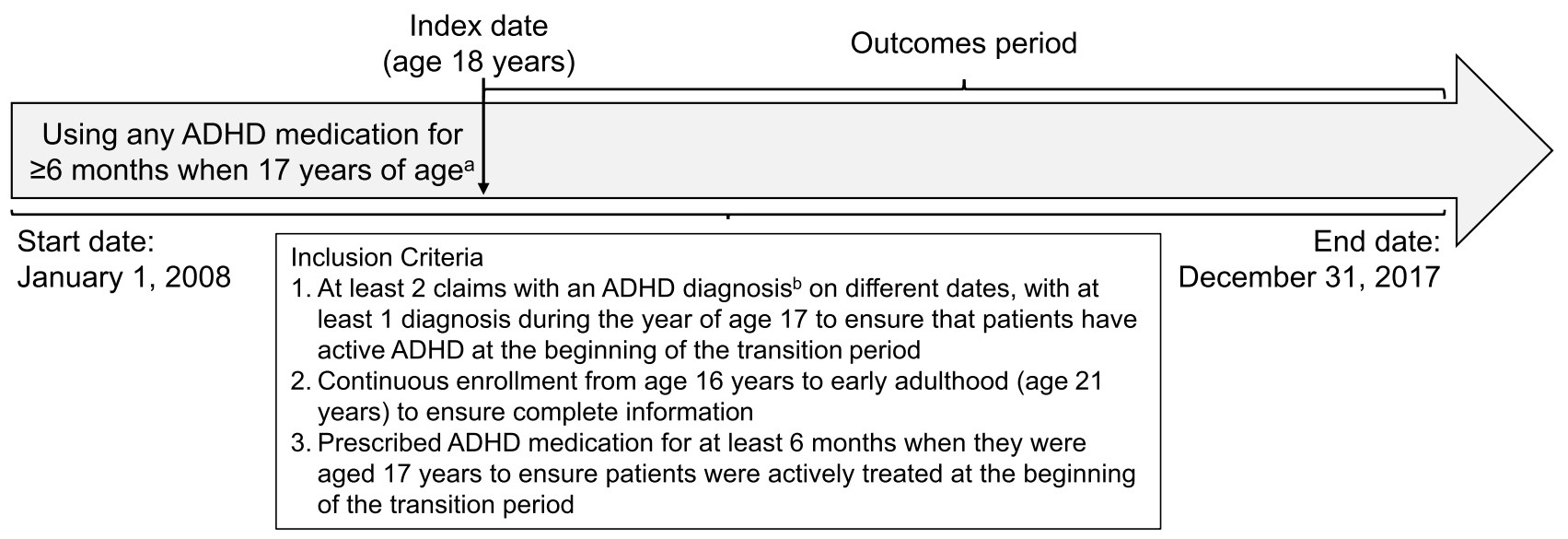

Fig. 1 Study design and inclusion criteria. ADHD attention-deficit/ hyperactivity disorder. ${ }^{\text {a }}$ Of all patients who met these criteria, $90 \%$

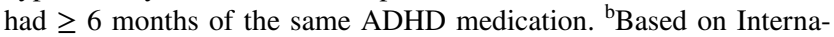

\subsection{Statistical Analyses}

Data analyses were conducted using SAS Version 9.4 (SAS Institute, Cary, NC, USA) or R Version 3.4.2. All analyses were conducted in the overall population and in subgroups of patients with ADHD with co-occurring psychiatric disorders and without co-occurring psychiatric disorders. Because this was a retrospective database study, a formal sample size calculation was not conducted.

Demographic and baseline characteristics are presented using descriptive statistics. Comparisons of ADHD pharmacotherapy were assessed longitudinally across age groups (16-17 vs $18-19$ vs $20-21$ years) in the overall population and in two ADHD subgroups (patients with co-occurring psychiatric disorders vs patients without co-occurring psychiatric disorders). Wilcoxon signed-rank tests were used to assess continuous variables, and McNemar tests were used to assess categorical variables. Within each age group, treatment differences between ADHD subgroups with vs without co-occurring psychiatric disorders were also assessed using Wilcoxon rank-sum tests and Chi-square tests. Kaplan-Meier analyses were used to assess the proportion of patients who discontinued, switched, or augmented their ADHD pharmacotherapy by the end of years 1,2, and 3 . These data are reported as the median (95\% confidence interval) time in years. For the assessment of treatment sequence, the top ten treatment sequences for patients aged 17-21 years are reported. In addition, an alluvial diagram is used to present the flow across treatments from year to year.

For HRU, the percentage of patients with one or more visits across HRU categories is reported for each age group. Healthcare resource utilization was compared among age groups in the overall population and in each ADHD subgroup using the Wilcoxon signed-rank tests for continuous tional Classification of Diseases, Ninth Revision, Clinical Modification, code $314.0 \mathrm{x}$ or International Classification of Diseases, Tenth Revision, Clinical Modification, code F90.x

variables and McNemar tests for categorical variables. Within each age group, differences between the ADHD co-occurring psychiatric disorder subgroups were assessed using Wilcoxon rank-sum tests for continuous variables and Chi-square tests for categorical variables.

\section{Results}

\subsection{Patient Disposition and Characteristics}

Of the 279,334 patients identified as having two or more claims with an ADHD diagnosis on different dates and having one or more diagnoses during the year of age 17 , 10,292 patients met study inclusion criteria (Fig. 2). Baseline demographic and clinical characteristics are summarized in Table 1. Most patients in the overall population were male and had co-occurring psychiatric disorders $(60.2 \%$ [6193/10,292]). Among patients with a co-occurring psychiatric disorder, the most frequently reported disorders (by $>5 \%$ of patients) were anxiety disorders (17.5\% [1081/6193]); trauma- and stressor-related disorders (14.9\% [924/6193]); depressive disorders (12.5\% [772/6193]); neurodevelopment disorders (7.3\% [449/6193]); disruptive, impulse control, and conduct disorders (6.6\% [406/6193]); and bipolar and related disorders (5.7\% [355/6193]). The largest percentage of patients in the overall population lived in the southern region of the USA and had a preferred provider organization type of insurance (Table 1).

\subsection{Treatment Use}

Significantly higher percentages of patients in the overall population received any ADHD pharmacotherapy, LA 
stimulant therapy, SA stimulant therapy, or non-stimulant therapy when aged 16-17 years compared with when aged 18-19 years or 20-21 years (Fig. 3). Similar patterns of statistically significant decreases in ADHD pharmacotherapy use were observed in both patients with co-occurring psychiatric disorders and patients without co-occurring psychiatric disorders (Table 2). Furthermore, statistically significant differences in ADHD pharmacotherapy use were observed between these subgroups (Table 2). When aged 16-17 years, a significantly lower percentage of patients with co-occurring psychiatric disorders used LA stimulants and significantly greater percentages used SA stimulants and non-stimulants compared with patients without cooccurring psychiatric disorders. When aged 18-19 years, a significantly lower percentage of patients with co-occurring psychiatric disorders used LA stimulants and a significantly greater percentage used non-stimulants compared with patients without co-occurring psychiatric disorders. When aged 20-21 years, a significantly greater percentage of patients with co-occurring psychiatric disorders used any ADHD pharmacotherapy, SA stimulants, and non-stimulants compared with patients without co-occurring psychiatric disorders.

In the overall population, with the exception of dextroamphetamine-amphetamine mix SA, statistically significant decreases in ADHD pharmacotherapy use with increasing age were observed across specific therapies (Table 3 ). Across all age groups, significantly greater percentages of patients with co-occurring psychiatric disorders used lisdexamfetamine, dextroamphetamine-amphetamine mix SA, and non-stimulants compared with patients without co-occurring psychiatric disorders (Table 3). When aged 16-17 years, statistically significantly greater percentages of patients with co-occurring psychiatric disorders used methylphenidate SA compared with patients without co-occurring psychiatric disorders (Table 3).When aged 20-21 years, statistically significantly greater percentages of patients with co-occurring psychiatric disorders used dextroamphetamine SA compared with patients without co-occurring psychiatric disorders (Table 3).

\subsection{Treatment Discontinuation}

The median (95\% CI) time to discontinuation of ADHD pharmacotherapy in the overall population was 2.94 (2.86-3.03) years when discontinuation was defined as a treatment lapse of $>60$ days and 3.78 (3.69-3.85) years when discontinuation was defined as a treatment lapse of $>90$ days. The median $(95 \% \mathrm{CI})$ time to discontinuation was longer in patients with vs without co-occurring psychiatric disorders when defining discontinuation as a treatment lapse of $>60$ days $(3.12[3.00,3.21]$ vs $2.72[2.59,2.83]$ years $)$ or $>90$ days $(3.89[3.76,4.01]$ vs $3.64[3.52,3.78]$ years $)$.

\subsection{Treatment Switching and Augmentation}

Among patients in the overall population using ADHD pharmacotherapy on the index date $(n=8424)$, the percentages of patients switching treatment within 60 days before or after the discontinuation of index treatment were $11.1 \%$ after 1 year, $17.8 \%$ after 2 years, and $22.5 \%$ after 3 years. The median time to treatment switching was not reached during the study period.

Compared with patients without co-occurring psychiatric disorders ( $n=3318$ ), patients with co-occurring psychiatric disorders $(n=5106)$ switched treatment at a higher rate after $1(12.7 \%$ vs $8.5 \%), 2$ (19.2\% vs $15.6 \%)$, and 3 (24.4\% vs $19.6 \%$ ) years; the median time to treatment switching was not reached during the study period for either subgroup.
Fig. 2 Participant disposition. $A D H D$ attention-deficit/hyperactivity disorder

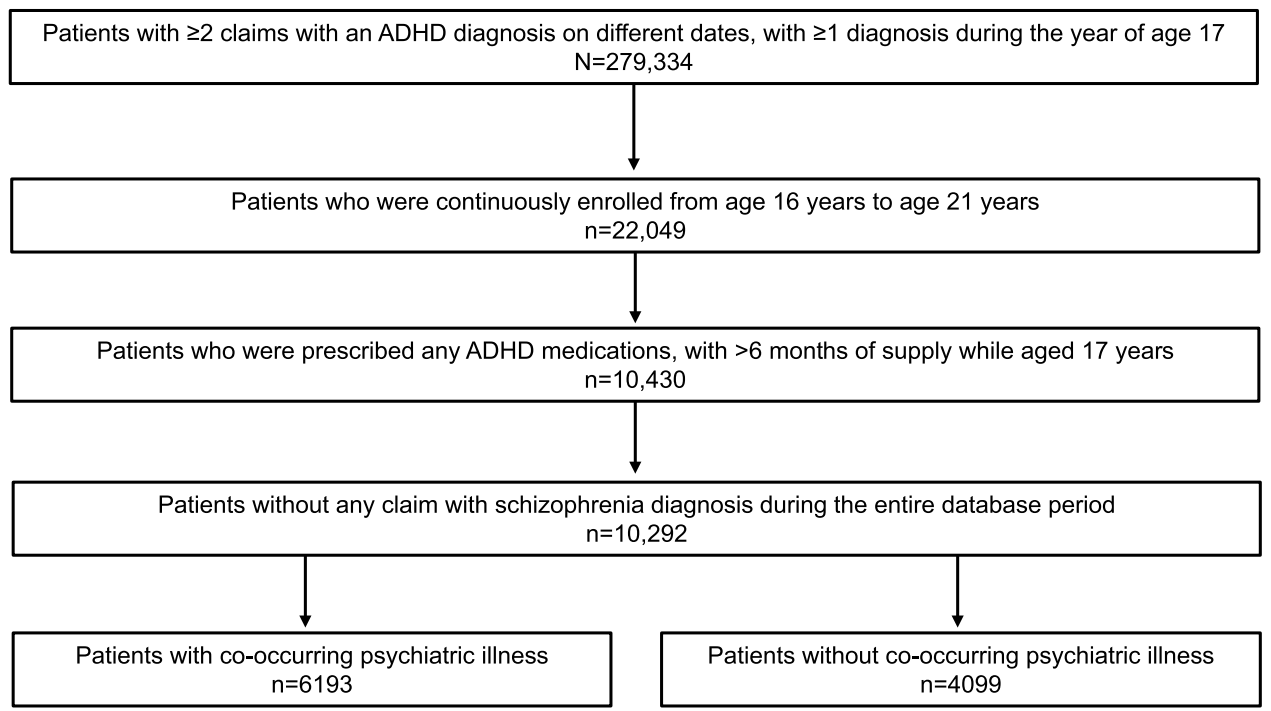


Table 1 Baseline demographic and clinical characteristics

\begin{tabular}{|c|c|c|c|}
\hline & \multirow[t]{2}{*}{ Total $(N=10,292)$} & \multicolumn{2}{|c|}{$\begin{array}{l}\text { Co-occurring psychiatric } \\
\text { disorder }\end{array}$} \\
\hline & & Yes $(n=6193)$ & No $(n=4099)$ \\
\hline \multicolumn{4}{|l|}{ Sex, $n(\%)$} \\
\hline Male & $6585(64.0)$ & $3714(60.0)$ & $2871(70.0)$ \\
\hline \multicolumn{4}{|c|}{ US region of residence, $n(\%)$} \\
\hline South & $4281(41.6)$ & 2391 (38.6) & $1890(46.1)$ \\
\hline North central & $2989(29.0)$ & $1862(30.1)$ & $1127(27.5)$ \\
\hline Northeast & $1684(16.4)$ & $1124(18.1)$ & $560(13.7)$ \\
\hline West & $1289(12.5)$ & $779(12.6)$ & $510(12.4)$ \\
\hline Unknown & $49(0.5)$ & $37(0.60)$ & $12(0.3)$ \\
\hline \multicolumn{4}{|c|}{ Insurance type, $n(\%)$} \\
\hline PPO & $5729(55.7)$ & $3473(56.1)$ & $2256(55.0)$ \\
\hline HMO & $1627(15.8)$ & $970(15.7)$ & $657(16.0)$ \\
\hline POS & $1161(11.3)$ & $686(11.1)$ & $475(11.6)$ \\
\hline CDHP & $937(9.1)$ & $543(8.8)$ & $394(9.6)$ \\
\hline HDHP & $363(3.5)$ & $224(3.6)$ & $139(3.4)$ \\
\hline COMP & $262(2.5)$ & $172(2.8)$ & $90(2.2)$ \\
\hline EPO & $100(1.0)$ & $60(1.0)$ & $40(1.0)$ \\
\hline Unknown & $84(0.8)$ & $47(0.8)$ & $37(0.9)$ \\
\hline CPOS & $29(0.3)$ & $18(0.3)$ & $11(0.3)$ \\
\hline \multicolumn{4}{|c|}{ Charlson Comorbidity Index ${ }^{a}$} \\
\hline Mean \pm SD & $0.10 \pm 0.35$ & $0.12 \pm 0.39$ & $0.07 \pm 0.28$ \\
\hline
\end{tabular}

Demographic characteristics were assessed as of the index date CDHP consumer-driven health plan, COMP comprehensive, CPOS capitated point-of-service, EPO exclusive provider organization, $H D H P$ health deductible health plan, $H M O$ health maintenance organization, $P O S$ non-capitated point-of-service, $P P O$ preferred provider organization, $S D$ standard deviation

${ }^{a}$ Charlson comorbidities were assessed during the year before a patient's 18th birthday
Of the 6802 patients in the overall population using ADHD pharmacotherapy by the index date and persisting with that treatment for $\geq 60$ days, the percentages of patients using an augmentation strategy were $16.7 \%$ after 1 year, $25.9 \%$ after 2 years, and $31.3 \%$ after 3 years. Greater percentages of patients with $(n=4095)$ vs without $(n=$ 2707) co-occurring psychiatric disorders used an augmentation strategy after 1 (21.0\% vs $10.0 \%), 2$ (31.3\% vs $17.2 \%)$, and 3 years $(38.5 \%$ vs $19.8 \%)$.

\subsection{Treatment Sequence}

The most frequently observed treatment sequences were starting and remaining on LA amphetamines or LA methylphenidates (Table 4). In six of the top ten sequences, patients were using an LA stimulant at 17 years of age but were no longer receiving pharmacotherapy by 21 years of age. Similar patterns were observed in analyses stratified by the presence or absence of co-occurring psychiatric disorders (Table 4).

Treatment flow in the overall population of patients aged 17-21 years is depicted in Fig. 4. Long-acting stimulants (amphetamines, 46.0\%; methylphenidates, 28.6\%) were the most frequently used ADHD pharmacotherapies in patients aged 17 years. With increases in age, there were decreases in the percentage of patients using LA amphetamines (18 years, $31.2 \%$; 19 years, $23.1 \%$; 20 years, $20.3 \%$; 21 years, $17.8 \%$ ) and LA methylphenidates (18 years, $17.7 \%$; 19 years, $12.0 \%$; 20 years, $9.5 \%$; 21 years, $7.8 \%)$. Similarly, the percentage of patients using non-stimulants decreased (17 years, $10.0 \% ; 18$ years, $7.2 \%$; 19 years, $5.2 \%$; 20 years, $4.5 \% ; 21$ years, $4.3 \%$ ) or using mixed therapy (17 years, $8.4 \% ; 18$ years, $4.1 \% ; 19$ years, $3.5 \%$; 20 years, $2.8 \% ; 21$ years, $2.3 \%$ ) also decreased as patients aged. The percentages of patients receiving no
Fig. 3 Attention-deficit/hyperactivity disorder (ADHD) pharmacotherapy use by age group in the overall population $(N=$ 10,292). $L A$ long-acting, $S A$ short-acting. ${ }^{*} p<0.05(16-17$ years vs $18-19$ years); ${ }^{\dagger} p<0.05$ (16-17 years vs $20-21$ years); ${ }^{\ddagger} p<0.05(18-19$ years vs $20-21$ years)

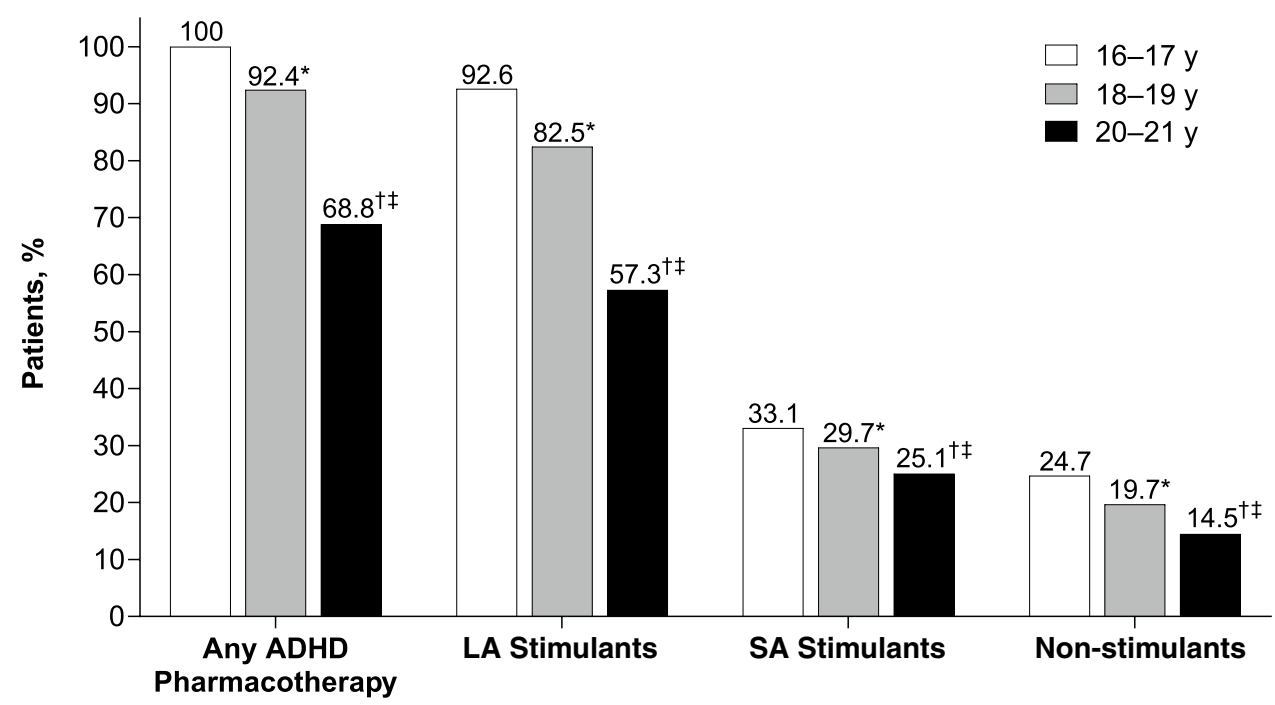


Table 2 ADHD pharmacotherapy use by age and presence or absence of co-occurring psychiatric disorders

\begin{tabular}{|c|c|c|c|c|c|c|}
\hline & \multicolumn{3}{|c|}{ Co-occurring psychiatric disorder $(n=6193)$} & \multicolumn{3}{|c|}{ No co-occurring psychiatric disorder $(n=4099)$} \\
\hline & $16-17 y$ & $18-19 y$ & $20-21$ y & $16-17 y$ & $18-19 y$ & $20-21 \mathrm{y}$ \\
\hline $\begin{array}{l}\text { Any ADHD pharmaco- } \\
\text { therapy, n (\%) }\end{array}$ & $6193(100.0)$ & $5726(92.5)^{*}$ & $4372(70.6)^{\dagger+\S}$ & $4099(100.0)$ & $3786(92.4)^{*}$ & $2713(66.2)^{\dagger+1}$ \\
\hline LA stimulants & $5697(92.0)^{\S}$ & $5048(81.5)^{* \S}$ & $3570(57.6)^{\dagger \neq}$ & $3833(93.5)$ & $3439(83.9)^{*}$ & $2324(56.7)^{\dagger *}$ \\
\hline SA stimulants & $2178(35.2)^{\S}$ & $1865(30.1)^{*}$ & $1628(26.3)^{\dagger \neq \S}$ & $1226(29.9)$ & $1187(29.0)$ & $951(23.2)^{\dagger \dagger}$ \\
\hline Non-stimulants & $1913(30.9)^{\S}$ & $1578(25.5)^{* \S}$ & $1227(19.8)^{\dagger \neq \S}$ & $628(15.3)$ & $445(10.9)^{*}$ & $263(6.4)^{\dagger \ddagger}$ \\
\hline
\end{tabular}

$A D H D$ attention-deficit/hyperactivity disorder, $L A$ long-acting, $S A$ short-acting, $y$ years

${ }^{*} p<0.05$ (16-17 y vs 18-19 y within psychiatric disorder subgroup), ${ }^{\dagger} p<0.05$ (16-17 y vs $20-21$ y within psychiatric disorder subgroup); ${ }^{\ddagger} p<0.05$ (18-19 y vs $20-21$ y within psychiatric disorder subgroup); ${ }^{\circledR} p<0.05$ (vs same age group without co-occurring psychiatric disorders)

Table 3 ADHD pharmacotherapy use by age and presence or absence of co-occurring psychiatric disorders

\begin{tabular}{|c|c|c|c|c|c|c|c|c|c|}
\hline \multirow[t]{2}{*}{$\begin{array}{l}\text { Treatment, } \\
n(\%)\end{array}$} & \multicolumn{3}{|c|}{ All patients $^{\mathrm{a}}(N=10,292)$} & \multicolumn{3}{|c|}{$\begin{array}{l}\text { With co-occurring psychiatric disorders } \\
(n=6193)\end{array}$} & \multicolumn{3}{|c|}{$\begin{array}{l}\text { Without co-occurring psychiatric disor- } \\
\text { ders }(n=4099)\end{array}$} \\
\hline & $16-17$ y & $18-19 y$ & $20-21$ y & $16-17 y$ & $18-19 y$ & $20-21$ y & $16-17 y$ & $18-19 y$ & $20-21$ y \\
\hline \multicolumn{10}{|l|}{ LA stimulants } \\
\hline MPH LA & 4009 (39.0) & $2876(27.9)^{*}$ & $1685(16.4)^{\dagger \dagger}$ & $2445(39.5)$ & $1689(27.3)^{*}$ & $1006(16.2)^{\dagger \dagger}$ & $1564(38.2)$ & $1187(29.0)^{*}$ & $679(16.6)^{\dagger \dagger}$ \\
\hline $\begin{array}{l}\text { Dex-MPH } \\
\text { LA }\end{array}$ & $932(9.1)$ & $668(6.5)^{*}$ & $378(3.7)^{\dagger \ddagger}$ & $588(9.5)$ & $426(6.9)^{*}$ & $239(3.9)^{\dagger \dagger}$ & $344(8.4)$ & $242(5.9)^{*}$ & $139(3.4)^{\dagger *}$ \\
\hline $\begin{array}{l}\text { Dextro- } \\
\text { AMP LA }\end{array}$ & $99(1.0)$ & $78(0.8)^{*}$ & $48(0.5)^{\dagger \hbar}$ & $57(0.9)$ & $50(0.8)$ & $32(0.5)^{\dagger \dagger}$ & $42(1.0)$ & $28(0.7)^{*}$ & $16(0.4)^{\dagger \neq}$ \\
\hline $\begin{array}{l}\text { Dextro- } \\
\text { AMP/ } \\
\text { AMP mix } \\
\text { LA }\end{array}$ & $3362(32.7)$ & $2838(27.6)^{*}$ & $2048(19.9)^{\dagger+}$ & $2024(32.7)$ & $1670(27.0)^{*}$ & $1252(20.2)^{\dagger \dagger}$ & $1338(32.6)$ & $1168(28.5)^{*}$ & $796(19.4)^{\dagger \dagger}$ \\
\hline $\begin{array}{l}\text { Lisdexam- } \\
\text { fetamine }\end{array}$ & $3653(35.5)$ & $3181(30.9)^{*}$ & $2349(22.8)^{\dagger+}$ & $2336(37.7)^{\S}$ & $2016(32.6)^{* \S}$ & $1469(23.7)^{\dagger \neq \S}$ & 1317 & $1165(28.4)^{*}$ & $880(21.5)^{\dagger}$ \\
\hline Overall & $9530(92.6)$ & $8487(82.5)^{*}$ & $5894(57.3)^{\dagger+}$ & $5697(92.0)^{\S}$ & $5048(81.5)^{* \S}$ & $3570(57.6)^{\dagger \dagger}$ & $3833(93.5)$ & $3439(83.9)^{*}$ & $2324(56.7)^{\dagger+}$ \\
\hline \multicolumn{10}{|l|}{ SA stimulants } \\
\hline MPH SA & 1223 (11.9) & $933(9.1)^{*}$ & $620(6.0)^{\dagger \dagger}$ & $784(12.7)^{\S}$ & $551(8.9)^{*}$ & $364(5.9)^{\dagger \ddagger}$ & 439 (10.7) & $382(9.3)^{*}$ & $256(6.2)^{\dagger \ddagger}$ \\
\hline $\begin{array}{l}\text { Dex-MPH } \\
\text { SA }\end{array}$ & $483(4.7)$ & $340(3.3)^{*}$ & $232(2.3)^{\dagger \dagger}$ & $308(5.0)$ & $209(3.4)^{*}$ & $141(2.3)^{\dagger ‡}$ & $175(4.3)$ & $131(3.2)^{*}$ & $91(2.2)^{\dagger \neq}$ \\
\hline $\begin{array}{l}\text { Dextro- } \\
\text { AMP SA }\end{array}$ & $148(1.4)$ & $108(1.0)^{*}$ & $87(0.9)^{\dagger+}$ & $101(1.6)$ & $73(1.2)^{*}$ & $66(1.1)^{\dagger \S}$ & $47(1.1)$ & $35(0.9)$ & $21(0.5)^{\dagger \neq}$ \\
\hline $\begin{array}{l}\text { Dextro- } \\
\text { AMP/ } \\
\text { AMP mix } \\
\text { SA }\end{array}$ & $1818(17.7)$ & 1839 (17.9) & $1796(17.4)$ & $1172(18.9)^{\S}$ & $1152(18.6)^{\S}$ & $1168(18.9)^{\S}$ & $646(15.8)$ & $687(16.8)$ & $628(15.3)^{\ddagger}$ \\
\hline Overall & 3404 (33.1) & $3052(29.6)^{*}$ & $2579(25.1)^{\dagger \dagger}$ & $2178(35.2)^{\S}$ & $1865(30.1)^{*}$ & $1628(26.3)^{\dagger \ddagger \S}$ & 1226 & $1187(29.0)$ & $951(23.2)^{\dagger+t}$ \\
\hline \multicolumn{10}{|c|}{ Non-stimulants } \\
\hline $\begin{array}{l}\text { Atomox- } \\
\text { etine }\end{array}$ & $1000(9.7)$ & $682(6.6)^{*}$ & $394(3.8)^{\dagger \dagger}$ & $676(10.9)^{\S}$ & $466(7.5)^{* \S}$ & $277(4.5)^{\dagger \dagger \S}$ & 324 (7.9) & $216(5.3)^{*}$ & $117(2.9)^{\dagger \neq}$ \\
\hline Clonidine & $553(5.4)$ & $394(3.8)^{*}$ & $272(2.6)^{\dagger \dagger}$ & $428(6.9)^{\S}$ & $321(5.2)^{* \S}$ & $234(3.8)^{\dagger \dagger \S}$ & $125(3.0)$ & $73(1.8)^{*}$ & $38(0.9)^{\dagger \neq}$ \\
\hline Guanfacine & $660(6.4)$ & $480(4.7)^{*}$ & $280(2.7)^{\dagger \dagger}$ & $530(8.6)^{\S}$ & $384(6.2)^{* \S}$ & $234(3.8)^{\dagger \dagger \S}$ & $130(3.2)$ & $96(2.3)^{*}$ & $46(1.1)^{\dagger \neq}$ \\
\hline Bupropion & $790(7.7)$ & $772(7.5)$ & $722(7.0)^{\dagger}$ & $675(10.9)^{\S}$ & $686(11.1)^{\S}$ & $644(10.4)^{\S}$ & $115(2.8)$ & $86(2.1)^{*}$ & $78(1.9)^{\dagger}$ \\
\hline Overall & $2541(24.7)$ & $2023(19.7)^{*}$ & $1490(14.5)^{\dagger \dagger}$ & $1913(30.9)^{\S}$ & $1578(25.5)^{* \S}$ & $1227(19.8)^{\dagger \neq \S}$ & $628(15.3)$ & $445(10.9)^{*}$ & $263(6.4)^{\dagger \neq}$ \\
\hline
\end{tabular}

$A D H D$ attention-deficit/hyperactivity disorder, $A M P$ amphetamine, Dex dexmethylphenidate, Dextro dextroamphetamine, $L A$ long-acting, $M P H$ methylphenidate, $S A$ short-acting, $y$ years

${ }^{*} p<0.05$ (16-17 y vs $18-19$ y within psychiatric disorder subgroup); ${ }^{\dagger} p<0.05$ (16-17 y vs $20-21$ y within psychiatric disorder subgroup); ${ }^{\ddagger} p<0.05$ (18-19 y vs $20-21$ y within psychiatric disorder subgroup); ${ }^{\S} p<0.05$ (vs same age group without co-occurring psychiatric disorders)

${ }^{a}$ Many patients used more than one type of ADHD pharmacotherapy in each 2-year period from ages 16 to 21 years, meaning that there is overlap in therapies and therefore the columns in this table do not sum to the total number of patients 
treatment increased (18 years, 9.4\%; 19 years, $25.8 \% ; 20$ years, $35.7 \%$; 21 years, $42.8 \%$ ) or having disrupted treatment decreased (18 years, $24.1 \%$; 19 years, $23.8 \% ; 20$ years, $20.2 \%$; 21 years, $17.9 \%$ ) from age 18 to 21 years. Low percentages $(2.3-2.5 \%)$ of patients aged $17-21$ years were categorized as using combination therapy. After transitioning to no treatment or disrupted treatment, small percentages of patients returned to pharmacotherapy (no treatment: $2.7-3.8 \%$ per year; disrupted treatment: $15.7-21.5 \%$ per year).

Findings in patients with and without co-occurring psychiatric disorders were consistent with those of the overall population (Table 5). The most frequently used ADHD pharmacotherapies in patients aged 17 years were LA amphetamines and LA methylphenidates in patients with and without co-occurring psychiatric disorders. Between these subgroups, there were comparable decreases in the percentages of patients using LA amphetamines and LA methylphenidates with increases in age. Similarly, the percentage of patients using non-stimulants and using mixed therapy decreased from age 17 to 21 years in both groups. In both groups, the percentages of patients receiving no treatment increased and the percentages of patients with disrupted treatment decreased from age 18 to 21 years. Comparably small percentages of patients were categorized as using combination therapy (patients with co-occurring psychiatric disorders, 2.4-2.8\%; patients without co-occurring psychiatric disorders, $1.9-2.1 \%$ ). After transitioning to no treatment or disrupted treatment, small percentages of patients returned to pharmacotherapy (patients with co-occurring psychiatric disorders: $3.5-4.0 \%$ per year for no treatment and $16.0-23.3 \%$ per year for disrupted treatment; patients without co-occurring psychiatric comorbidities: $1.7-3.5 \%$ per year for no treatment and $15.4-20.9 \%$ per year for disrupted treatment).

\subsection{Healthcare Resource Utilization}

In the overall population, statistically significant decreases in all-cause inpatient and outpatient visits (all-cause, pediatrician, psychiatrist, ADHD-related pediatrician, ADHDrelated non-pediatrician, and mood disorder related) visits were observed with increasing age (Table 6). In contrast, a statistically significant increase in PCP visits was observed as patients aged. Patients with and without co-occurring psychiatric disorders exhibited patterns similar to the overall population, except that all-cause ED visits significantly increased with age in patients with co-occurring psychiatric disorders but did not increase in patients without co-occurring psychiatric disorders (Table 6).

The percentage of patients having one or more all-cause inpatient visit, all-cause ED visit, or outpatient visit (PCP, psychiatrist, non-pediatrician ADHD related, and mood disorder related) was significantly greater in patients with vs without co-occurring psychiatric disorders across all ages; at ages 18-19 and 20-21 years, the percentage of patients with co-occurring psychiatric disorders having one or more all-cause outpatient visit was significantly greater than in patients without co-occurring psychiatric disorders (Table 6). The percentage of patients having one or more ADHD-related visit or ADHD-related pediatrician visit was significantly lower in those with vs without co-occurring psychiatric disorders across all ages but was significantly greater for ADHD-related non-pediatrician visits (Table 6).

\section{Discussion}

The primary finding of this study is that as patients with ADHD transitioned from adolescence to early adulthood, the likelihood of discontinuing or experiencing disruptions in ADHD pharmacotherapy increased. Importantly, after discontinuing ADHD pharmacotherapy or having a treatment disruption, patients rarely reinitiated treatment. In addition, HRU also decreased as patients aged, with the exception of outpatient primary care visits, which increased with age. Although the observed patterns of ADHD pharmacotherapy use and HRU in the overall population were generally similar in subgroups of patients with vs without co-occurring psychiatric disorders, some differences were observed. Patients with co-occurring psychiatric disorders remained on ADHD pharmacotherapy longer and switched or augmented their pharmacotherapy more frequently than patients without co-occurring psychiatric comorbidities. The most frequently used medication class in both subgroups was LA stimulants, but across all age groups, greater percentages of patients with co-occurring psychiatric disorders used lisdexamfetamine, dextroamphetamine-amphetamine mix SA, and non-stimulants than patients without co-occurring psychiatric disorders. Last, patients with co-occurring psychiatric disorders generally exhibited greater levels of HRU than patients without co-occurring psychiatric disorders, with the exception of pediatrician visits and ADHD-related (any and pediatrician) visits, which were lower in patients with co-occurring psychiatric disorders.

The observed increases in discontinuation and disruption of ADHD pharmacotherapy as patients transitioned from adolescence to early adulthood are consistent with previously published findings [34]. In a retrospective cohort study, among patients who started treatment for ADHD between the ages of 6-12 years, $61.5 \%$ discontinued treatment before their 18th birthday [34]. Several factors have been suggested to contribute to treatment discontinuation during this period of transition, including inadequate health insurance coverage, inadequate clinical training and services, lack of transition planning and the abruptness of the 
Table 4 Top ten ADHD treatment sequences by age and presence or absence of co-occurring psychiatric disorders

\begin{tabular}{|c|c|c|c|c|c|c|}
\hline \multicolumn{2}{|c|}{ Frequency, $n(\%)$} & \multicolumn{5}{|l|}{ Patient age, y } \\
\hline & & 17 & 18 & 19 & 20 & 21 \\
\hline \multicolumn{7}{|c|}{ All patients $(N=10,292)$} \\
\hline 1 & $924(9.0)$ & LA amphetamines & LA amphetamines & LA amphetamines & LA amphetamines & LA amphetamines \\
\hline 2 & $519(5.0)$ & LA methylphenidates & LA methylphenidates & LA methylphenidates & LA methylphenidates & LA methylphenidates \\
\hline 3 & $410(4.0)$ & LA amphetamines & Disrupted treatment & No treatment & No treatment & No treatment \\
\hline 4 & $283(2.7)$ & LA methylphenidates & Disrupted treatment & No treatment & No treatment & No treatment \\
\hline 5 & $239(2.3)$ & LA amphetamines & No treatment & No treatment & No treatment & No treatment \\
\hline 6 & $203(2.0)$ & LA amphetamines & LA amphetamines & Disrupted treatment & No treatment & No treatment \\
\hline 7 & $185(1.8)$ & LA methylphenidates & No treatment & No treatment & No treatment & No treatment \\
\hline 8 & $166(1.6)$ & LA amphetamines & LA amphetamines & No treatment & No treatment & No treatment \\
\hline 9 & $160(1.6)$ & Non-stimulants & Non-stimulants & Non-stimulants & Non-stimulants & Non-stimulants \\
\hline 10 & $158(1.5)$ & LA amphetamines & LA amphetamines & LA amphetamines & LA amphetamines & Disrupted treatment \\
\hline \multicolumn{7}{|c|}{ With co-occurring psychiatric disorders $(n=6193)$} \\
\hline 1 & $541(8.7)$ & LA amphetamines & LA amphetamines & LA amphetamines & LA amphetamines & LA amphetamines \\
\hline 2 & $293(4.7)$ & LA methylphenidates & LA methylphenidates & LA methylphenidates & LA methylphenidates & LA methylphenidates \\
\hline 3 & $215(3.5)$ & LA amphetamines & Disrupted treatment & No treatment & No treatment & No treatment \\
\hline 4 & $135(2.2)$ & LA amphetamines & No treatment & No treatment & No treatment & No treatment \\
\hline 5 & $132(2.1)$ & LA methylphenidates & Disrupted treatment & No treatment & No treatment & No treatment \\
\hline 6 & $118(1.9)$ & LA amphetamines & LA amphetamines & Disrupted treatment & No treatment & No treatment \\
\hline 7 & $115(1.9)$ & Non-stimulants & Non-stimulants & Non-stimulants & Non-stimulants & Non-stimulants \\
\hline 8 & $100(1.6)$ & LA methylphenidates & No treatment & No treatment & No treatment & No treatment \\
\hline 9 & $86(1.4)$ & LA amphetamines & LA amphetamines & LA amphetamines & LA amphetamines & Disrupted treatment \\
\hline 10 & $84(1.4)$ & LA amphetamines & LA amphetamines & No treatment & No treatment & No treatment \\
\hline \multicolumn{7}{|c|}{ Without co-occurring psychiatric disorders $(n=4099)$} \\
\hline 1 & $383(9.3)$ & LA amphetamines & LA amphetamines & LA amphetamines & LA amphetamines & LA amphetamines \\
\hline 2 & $226(5.5)$ & LA methylphenidates & LA methylphenidates & LA methylphenidates & LA methylphenidates & LA methylphenidates \\
\hline 3 & $195(4.8)$ & LA amphetamines & Disrupted treatment & No treatment & No treatment & No treatment \\
\hline 4 & $151(3.7)$ & LA methylphenidates & Disrupted treatment & No treatment & No treatment & No treatment \\
\hline 5 & $104(2.5)$ & LA amphetamines & No treatment & No treatment & No treatment & No treatment \\
\hline 6 & $85(2.1)$ & LA amphetamines & LA amphetamines & Disrupted treatment & No treatment & No treatment \\
\hline 7 & $85(2.1)$ & LA methylphenidates & No treatment & No treatment & No treatment & No treatment \\
\hline 8 & $82(2.0)$ & LA amphetamines & LA amphetamines & No treatment & No treatment & No treatment \\
\hline 9 & $72(1.8)$ & LA amphetamines & LA amphetamines & LA amphetamines & LA amphetamines & Disrupted treatment \\
\hline 10 & $67(1.6)$ & LA methylphenidates & LA methylphenidates & Disrupted treatment & No treatment & No treatment \\
\hline
\end{tabular}

During each year, patients were categorized as the following: no treatment (patient had no drug supply); disrupted treatment (patient had some drug supply but totaling $\leq 6$ months of supply across all drug classes); specific drug class (LA amphetamine, LA methylphenidate, non-stimulant; patient had one or more drug class that individually had $\geq 6$ months of supplies; patients were categorized according to the drug class with the most days of supply)

$A D H D$ attention-deficit/hyperactivity disorder, $L A$ long-acting, $y$ years

transition, poor disease management owing to independent living during transition to college, poor disease insight, and patient/family resistance $[20,35,36]$. Recently, meta-analytic evidence showed that ADHD is significantly associated with an increased risk of unintentional physical injuries in children and adolescents, and pharmacologic interventions had a protective effect $[15,37]$. Taken together, these findings emphasize the need for having an increased focus on the management of ADHD during the transition period from adolescence to adulthood.

Consistent with the observed age-related reductions in ADHD pharmacotherapy use, reductions across multiple HRU categories were observed. In the overall population, the percentage of patients with one or more all-cause inpatient visits or all-cause outpatient visits declined as patients aged. However, when outpatient visits were examined in more detail, PCP visits were found to increase with age. The 


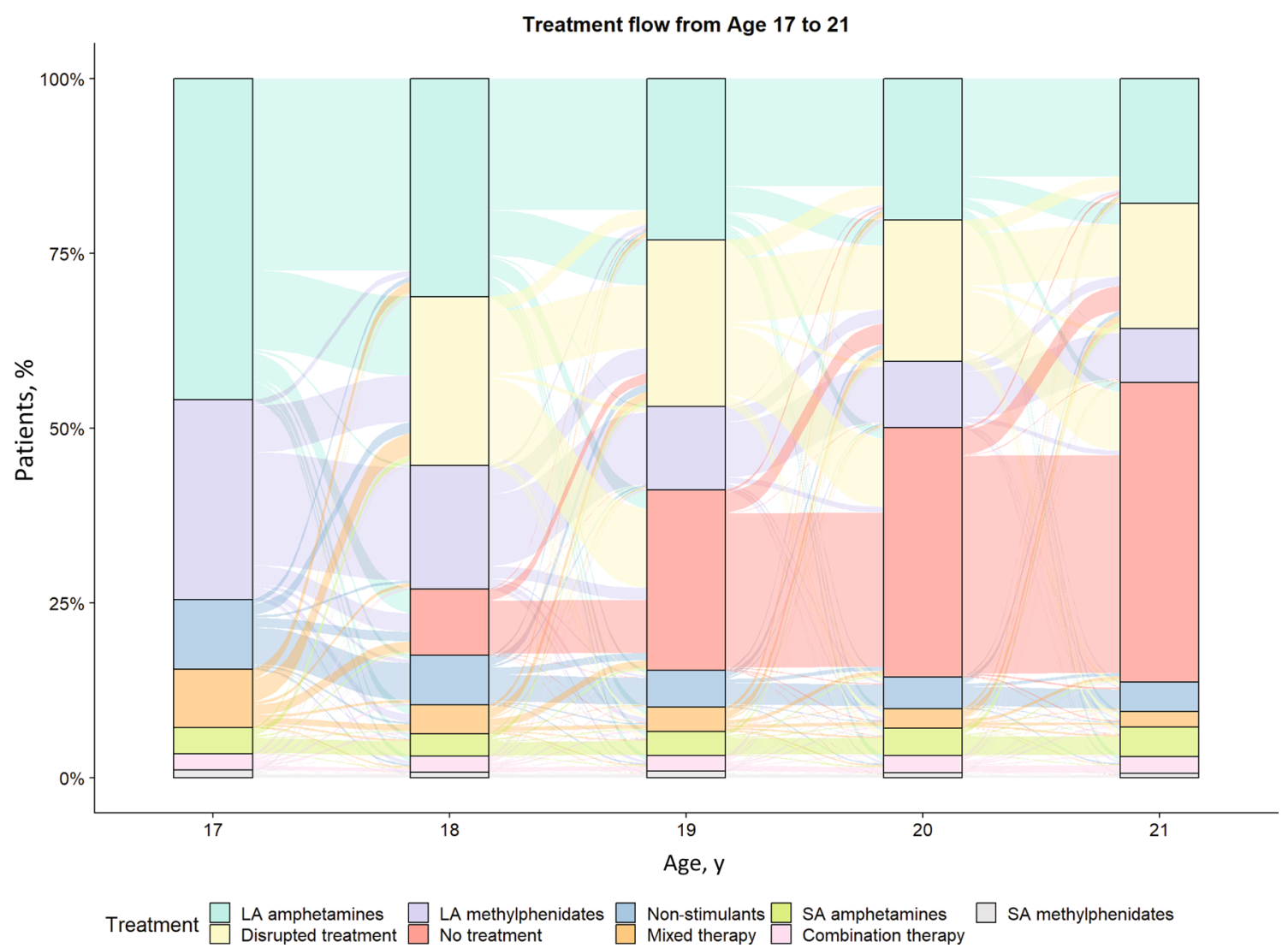

Fig. 4 Treatment sequence flow in patients with attention-deficit/ hyperactivity disorder (ADHD) from age 17 to 21 years in the overall population $(N=10,292)$. $L A$ long-acting, $S A$ short-acting. During each year, patients were categorized as the following: no treatment (patient had no drug supply); disrupted treatment (patient had some drug supply but totaling $\leq 6$ months of supply across all drug classes); specific drug class (LA or SA amphetamine, LA or
SA methylphenidate, non-stimulant; patient had $\geq 1$ drug class that individually had $\geq 6$ months of supplies; patients were categorized according to the drug class with the most days of supply); mixed therapy (patient had $\geq 6$ months of medication supply but not for any individual drug class); combination therapy (patient had $\geq 6$ months of supply for $\geq 1$ drug class with multiple drug classes tied for the highest number of days of supply) observed decreases in psychiatrist visits and ADHD-related visits with age in this study are consistent with findings from a published literature review that reported a $45 \%$ reduction in mental health service use in the USA by the age of 16 years and respective reductions of $24 \%$ and $>60 \%$ in the UK by the ages of 16 and 18 years [38]. In light of the widespread decreases in HRU (including decreased psychiatrist and ADHD-related visits) observed in this study, the increases in PCP visits are of interest but are not unexpected, as patients make the transition from pediatric to adult care. These data emphasize the need for PCPs to be knowledgeable regarding the persistence of ADHD into adulthood because they are likely to encounter young adults with ADHD who have recently transitioned from pediatric care.

Patients with co-occurring psychiatric disorders remained on ADHD pharmacotherapy longer and switched or augmented their pharmacotherapy more frequently. The finding that patients with co-occurring psychiatric disorders remain on treatment longer is not consistent with reports indicating that higher comorbidity burden in individuals with ADHD can negatively influence the maintenance of ADHD treatment [30]. The reason for this apparent discrepancy is unknown, but it could be speculated that the increased overall HRU in patients with co-occurring psychiatric disorders (including psychiatrist visits and ADHD-related non-pediatrician visits) mitigated the disruption and/or discontinuation of ADHD pharmacotherapy in these patients. The persistent use of psychiatric and ADHD-related nonpediatrician services could also account for the increased use of switching and augmentation strategies and suggests that managing ADHD was complicated by the presence of other psychiatric comorbidities. It could also be speculated that some level of treatment discontinuation is the result of symptom resolution, resulting in a decreased need for ADHD pharmacotherapy. In patients with co-occurring psychiatric disorders, the persistence of symptoms not related to ADHD could make it more difficult to determine if ADHD symptoms are resolving to a level that no longer requires 


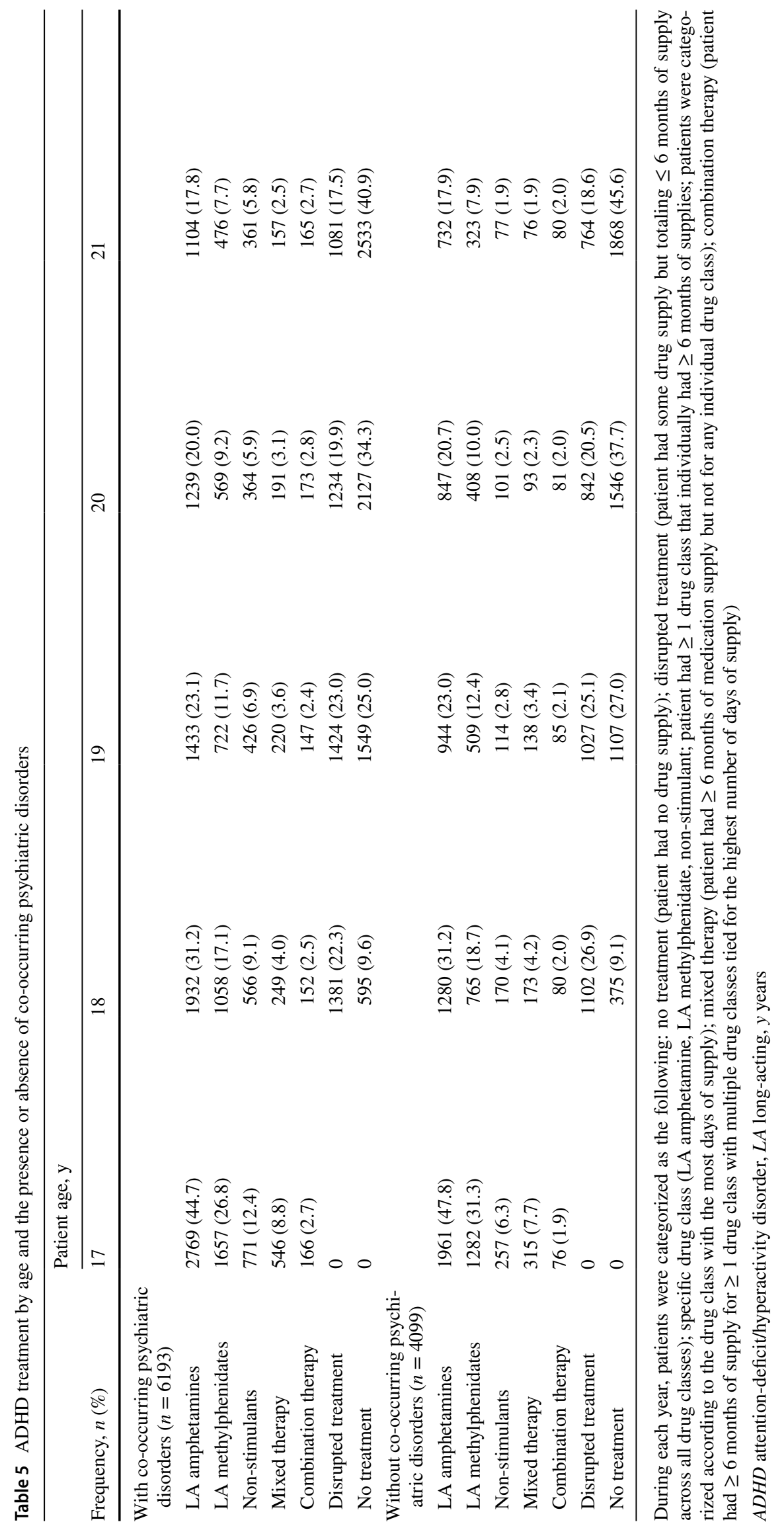




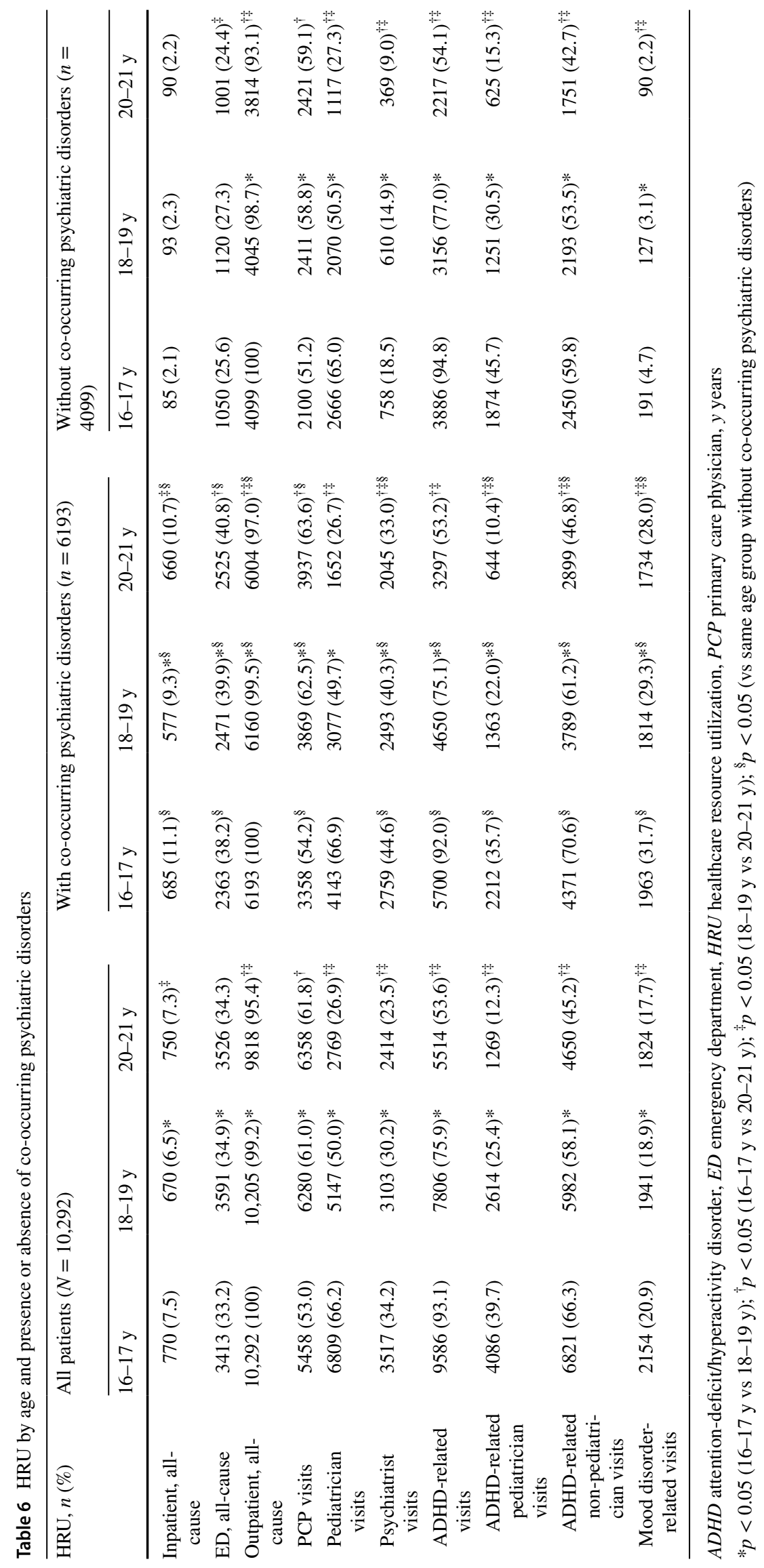


medication, resulting in more persistent ADHD pharmacotherapy use.

In regard to pharmacotherapy use, the most frequently used medication class in both groups was LA stimulants, but greater percentages of patients with co-occurring psychiatric disorders used lisdexamfetamine, dextroamphetamineamphetamine mix SA, and non-stimulants. It is not clear why lisdexamfetamine and dextroamphetamine-amphetamine mix SA would be used more frequently in patients with co-occurring psychiatric disorders. However, the increased use of non-stimulants might be related to the fact that they are recommended as first-line treatment in patients with intolerance or insufficient response to stimulant medications $[11,12]$.

Greater percentages of patients with co-occurring psychiatric disorders utilized healthcare resources compared with patients without co-occurring psychiatric disorders. Overall, these findings are consistent with previous reports that the presence of a co-occurring disorder increases HRU and costs in adults diagnosed with ADHD [25, 32]. In an electronic medical records analysis, the presence of multiple psychiatric disorders among adults diagnosed with ADHD resulted in an increased number of outpatient, inpatient, and ED visits and a decrease in behavioral therapy [32]. Interestingly, a lower percentage of patients with co-occurring psychiatric disorders had ADHD-related pediatrician visits compared with patients without co-occurring psychiatric disorders. In light of the greater percentages of patients with co-occurring psychiatric disorders who utilized psychiatrists and ADHDrelated non-pediatricians, this suggests that patients with co-occurring psychiatric disorders shifted away from using pediatricians and toward using specialists for the management of their psychiatric disorders, including ADHD.

These data should be considered in light of certain limitations. First, this was a retrospective observational study, thus causal relationships cannot be inferred from the data. Second, the study cohorts were defined using diagnostic codes that may be affected by misclassification because of the nature of data source. Third, treatment patterns were defined using prespecified algorithms, which may not reflect the physician's intent. Additionally, information regarding the underlying reasons for changes in treatment patterns is not available. Some discontinuations in treatment for ADHD pharmacotherapy are likely owing to the loss of insurance coverage or the perceived resolution of ADHD symptoms. In addition, the study results are only generalizable to commercially insured populations in the USA and may not be representative of the general population of patients diagnosed with ADHD. Finally, to be included in the study, patients must have remained on their parent's insurance beyond age 18 years. Therefore, these data may not generalize to all patients with ADHD transitioning to adulthood.

\section{Conclusions}

As patients with ADHD transitioned from adolescence to early adulthood, the percentage using ADHD pharmacotherapy decreased, and the proportion experiencing treatment disruptions or discontinuing treatment increased. After experiencing a treatment disruption or discontinuing treatment, patients rarely reinitiated treatment. These findings emphasize the need for increased focus on the management of patients with ADHD during this transition period to mitigate the disruption/discontinuation of treatment, which can result in negative outcomes [20, 21]. A concurrent decline in HRU was also observed as patients transitioned into early adulthood, with the exception of PCP visits, which increased. In light of the increased percentage of patients using PCPs as they age, it is critical that these physicians be knowledgeable regarding the persistence of ADHD into adulthood. Last, the pattern of changes in ADHD pharmacotherapy use during the transition to adulthood was generally comparable between patients with vs without co-occurring psychiatric disorders, but some differences were observed. Patients with co-occurring psychiatric disorders remained on ADHD pharmacotherapy for longer periods, switched and augmented their therapy more often, and had greater HRU. These findings emphasize that the presence of co-occurring psychiatric comorbidities can directly or indirectly influence the course of ADHD treatment, which emphasizes the need to closely monitor these patients.

Acknowledgements Under the direction of the authors, Sonia Mohinta, $\mathrm{PhD}$, and Craig Slawecki, PhD, employees of ICON (North Wales, PA, USA), provided writing assistance for this manuscript. Funding to ICON for support in writing this manuscript was provided by Takeda Pharmaceuticals USA, Inc. (Lexington, MA, USA).

\section{Declarations}

Funding This study was supported by funding from Shire Development LLC, a member of the Takeda group of companies, Lexington, MA, USA. Open access was funded by Shire Development LLC, a member of the Takeda group of companies, Lexington, MA, USA.

Conflict of interest Sepehr Farahbakhshian, Daniel S. Barczak, and William Spalding are employees of Takeda Pharmaceuticals USA and hold Takeda stock. Simerpal K. Gill is an employee of Takeda Canada Inc. and holds Takeda stock. Rajeev Ayyagari, Wenxi Tang, Thomas Kulalert, and Madeline Jenkins are employees of Analysis Group, Inc., which was funded by Shire, a member of the Takeda group of companies, to conduct this research.

Ethics approval This study did not involve prospective data collection; therefore, institutional review board approval was not required. Patient data were deidentified in accordance with the patient confidentiality requirements of the Health Insurance Portability and Accountability Act of 1996. A summary of the final study report was provided to the appropriate regulatory bodies in compliance with appropriate guidelines. 
Consent to participate Not applicable.

Consent for publication Not applicable.

Availability of data and material The data that support the findings of this study are available from the IBM ${ }^{\circledR}$ MarketScan ${ }^{\circledR}$ Commercial Databases, but restrictions apply to the availability of these data, which were used under license for the current study, and so are not publicly available.

Authors' contributions All authors contributed to the development of the manuscript, reviewed it at each stage of development, and approved the final draft for submission to CNS Drugs.

Open Access This article is licensed under a Creative Commons Attribution-NonCommercial 4.0 International License, which permits any non-commercial use, sharing, adaptation, distribution and reproduction in any medium or format, as long as you give appropriate credit to the original author(s) and the source, provide a link to the Creative Commons licence, and indicate if changes were made. The images or other third party material in this article are included in the article's Creative Commons licence, unless indicated otherwise in a credit line to the material. If material is not included in the article's Creative Commons licence and your intended use is not permitted by statutory regulation or exceeds the permitted use, you will need to obtain permission directly from the copyright holder. To view a copy of this licence, visit http://creativecommons.org/licenses/by-nc/4.0/.

\section{References}

1. Centers for Disease Control and Prevention. Attention-deficit/ hyperactivity disorder fact sheet. 2020. https://www.cdc.gov/ ncbddd/adhd/facts.html. Accessed 11 May 2020.

2. Thomas R, Sanders S, Doust J, et al. Prevalence of attention-deficit/hyperactivity disorder: a systematic review and meta-analysis. Pediatrics. 2015;135:e994-1001.

3. Lara C, Fayyad J, de Graaf R, et al. Childhood predictors of adult attention-deficit/hyperactivity disorder: results from the World Health Organization World Mental Health Survey Initiative. Biol Psychiatry. 2009;65:46-54.

4. Kessler RC, Green JG, Adler LA, et al. Structure and diagnosis of adult attention-deficit/hyperactivity disorder: analysis of expanded symptom criteria from the Adult ADHD Clinical Diagnostic Scale. Arch Gen Psychiatry. 2010;67:1168-78.

5. Kessler RC, Adler LA, Barkley R, et al. Patterns and predictors of attention-deficit/hyperactivity disorder persistence into adulthood: results from the National Comorbidity Survey Replication. Biol Psychiatry. 2005;57:1442-51.

6. Cortese S. The neurobiology and genetics of attention-deficit/ hyperactivity disorder (ADHD): what every clinician should know. Eur J Paediatr Neurol. 2012;16:422-33.

7. Blum K, Chen AL, Braverman ER, et al. Attention-deficit-hyperactivity disorder and reward deficiency syndrome. Neuropsychiatr Dis Treat. 2008;4:893-918.

8. Hoogman M, Bralten J, Hibar DP, et al. Subcortical brain volume differences in participants with attention deficit hyperactivity disorder in children and adults: a cross-sectional mega-analysis. Lancet Psychiatry. 2017;4:310-9.

9. Faraone SV. The pharmacology of amphetamine and methylphenidate: relevance to the neurobiology of attention-deficit/hyperactivity disorder and other psychiatric comorbidities. Neurosci Biobehav Rev. 2018;87:255-70.
10. Wolraich M, Subcommittee on Attention-Deficit/Hyperactivity Disorder, Steering Committee on Quality Improvement and Management, et al. ADHD: clinical practice guideline for the diagnosis, evaluation, and treatment of attention-deficit/hyperactivity disorder in children and adolescents. Pediatrics. 2011;128:1007-22.

11. National Institute for Health and Care Excellence. Attention deficit hyperactivity disorder: diagnosis and management. NICE guideline (NG87). London: 2018.

12. Canadian Attention Deficit Hyperactivity Disorder Resource Alliance (CADDRA). Canadian ADHD practice guidelines, 4.1 Edition. Toronto: CADDRA; 2020.

13. Cortese S, Adamo N, Del Giovane C, et al. Comparative efficacy and tolerability of medications for attention-deficit hyperactivity disorder in children, adolescents, and adults: a systematic review and network meta-analysis. Lancet Psychiatry. 2018;5:727-38.

14. Cortese S. Pharmacologic treatment of attention deficit-hyperactivity disorder. N Engl J Med. 2020;383:1050-6.

15. Ruiz-Goikoetxea M, Cortese S, Aznarez-Sanado M, et al. Risk of unintentional injuries in children and adolescents with ADHD and the impact of ADHD medications: a systematic review and meta-analysis. Neurosci Biobehav Rev. 2018;84:63-71.

16. Kessler RC, Adler L, Barkley R, et al. The prevalence and correlates of adult ADHD in the United States: results from the National Comorbidity Survey Replication. Am J Psychiatry. 2006;163:716-23.

17. Pawaskar M, Witt EA, Ming EE, et al. Estimating the prevalence of DSM-5 attention deficit/hyperactivity disorder (ADHD) in a community sample [P6-003]. American Psychiatric Association Annual Meeting; 16-20 May 2015; Toronto (ON).

18. Kessler RC, Berglund P, Chiu WT, et al. The US National Comorbidity Survey Replication (NCS-R): design and field procedures. Int J Methods Psychiatr Res. 2004;13:69-92.

19. Gajria K, Lu M, Sikirica V, et al. Adherence, persistence, and medication discontinuation in patients with attention-deficit/ hyperactivity disorder: a systematic literature review. Neuropsychiatr Dis Treat. 2014;10:1543-69.

20. Schaefer MR, Rawlinson AR, Wagoner ST, et al. Adherence to attention-deficit/hyperactivity disorder medication during the transition to college. J Adolesc Health. 2017;60:706-13.

21. Hechtman L, Swanson JM, Sibley MH, et al. Functional adult outcomes 16 years after childhood diagnosis of attention-deficit/ hyperactivity disorder: MTA results. J Am Acad Child Adolesc Psychiatry. 2016;55:945-52.

22. Eklund H, Cadman T, Findon J, et al. Clinical service use as people with attention deficit hyperactivity disorder transition into adolescence and adulthood: a prospective longitudinal study. BMC Health Serv Res. 2016;16:248.

23. Burke R, Spoerri M, Price A, et al. Survey of primary care pediatricians on the transition and transfer of adolescents to adult health care. Clin Pediatr (Phila). 2008;47:347-54.

24. McCarthy S, Asherson P, Coghill D, et al. Attention-deficit hyperactivity disorder: treatment discontinuation in adolescents and young adults. Br J Psychiatry. 2009;194:273-7.

25. Libutzki B, Ludwig S, May M, et al. Direct medical costs of ADHD and its comorbid conditions on basis of a claims data analysis. Eur Psychiatry. 2019;58:38-44.

26. Mohammadi MR, Zarafshan H, Khaleghi A, et al. Prevalence of ADHD and its comorbidities in a population-based sample. $\mathrm{J}$ Atten Disord. 2019. https://doi.org/10.1177/1087054719886372 (Epub ahead of print).

27. Chen Q, Hartman CA, Haavik J, et al. Common psychiatric and metabolic comorbidity of adult attention-deficit/hyperactivity disorder: a population-based cross-sectional study. PLoS ONE. 2018;13:e0204516. 
28. Kirino E, Imagawa H, Goto T, et al. Sociodemographics, comorbidities, healthcare utilization and work productivity in Japanese patients with adult ADHD. PLoS ONE. 2015;10:e132233.

29. Katzman MA, Bilkey TS, Chokka PR, et al. Adult ADHD and comorbid disorders: clinical implications of a dimensional approach. BMC Psychiatry. 2017;17:302.

30. Khan MU, Aslani P. A review of factors influencing the three phases of medication adherence in people with attention-deficit/hyperactivity disorder. J Child Adolesc Psychopharmacol. 2019;29:398-418.

31. Du Rietz E, Jangmo A, Kuja-Halkola R, et al. Trajectories of healthcare utilization and costs of psychiatric and somatic multimorbidity in adults with childhood ADHD: a prospective registerbased study. J Child Psychol Psychiatry. 2020. https://doi.org/10. 1111/jcpp.13206.

32. Kawatkar AA, Knight TK, Moss RA, et al. Impact of mental health comorbidities on health care utilization and expenditure in a large US managed care adult population with ADHD. Value Health. 2014;17:661-8.

33. Quan H, Sundararajan V, Halfon P, et al. Coding algorithms for defining comorbidities in ICD-9-CM and ICD-10 administrative data. Med Care. 2005;43:1130-9.
34. McCarthy S, Wilton L, Murray ML, et al. Persistence of pharmacological treatment into adulthood, in UK primary care, for ADHD patients who started treatment in childhood or adolescence. BMC Psychiatry. 2012;12:219.

35. Montano CB, Young J. Discontinuity in the transition from pediatric to adult health care for patients with attention-deficit/hyperactivity disorder. Postgrad Med. 2012;124:23-32.

36. Robb A, Findling RL. Challenges in the transition of care for adolescents with attention-deficit/hyperactivity disorder. Postgrad Med. 2013;125:131-40.

37. Ghirardi L, Larsson H, Chang Z, et al. Attention-deficit/hyperactivity disorder medication and unintentional injuries in children and adolescents. J Am Acad Child Adolesc Psychiatry. 2020;59:944-51.

38. Singh SP, Tuomainen H. Transition from child to adult mental health services: needs, barriers, experiences and new models of care. World Psychiatry. 2015;14:358-61. 